\title{
A novel repair for comminuted fractures in metacarpals: Combining cyanoacrylate with demineralized bone matrix
}

\author{
Michael Stephanides ${ }^{1,2}$, Angel Farinas ${ }^{2}$, S Peir Johnson ${ }^{2}$, Matthew Thayer ${ }^{3}$ and Wesley Thayer ${ }^{2,4 *}$ \\ ${ }^{1}$ Meharry Medical School, Nashville, TN, USA \\ ${ }^{2}$ Department of Plastic Surgery, Vanderbilt University Medical Center, Nashville, TN, USA \\ ${ }^{3}$ Montgomery Bell Academy, Nashville, TN, USA \\ ${ }^{4}$ Department of Biomedical Engineering, Vanderbilt University, Nashville, TN, USA
}

\begin{abstract}
Background: Comminuted fractures of the metacarpals are both extremely painful and difficult for surgeons to repair. A strong, fast acting repair must be made to realign minute fragments of bone properly. Cyanoacrylate and demineralized bone matrix (DBM) have proven effective individually for certain repairs, but the efficacy of a combined treatment has not been confirmed. The purpose of this study is to test the strength of the repair when using a combined treatment of cyanoacrylate mixed with DBM in comminuted fractures.

Methods: Sixteen metacarpals (2nd-5th) were collected from cadavers and fractured into three pieces at 30-degree angles to simulate a comminuted fracture. Three treatment groups were assessed: repair with cyanoacrylate glue $(n=4)$, repair with cyanoacrylate and DBM combined ( $n=4)$, and repair using titanium plates and screws $(\mathrm{n}=8)$. Bones were pulled apart until repair failure with an Instron 5542 machine and the load at break (N) was recorded for each sample. IBM SPSS Statistics 24 was used to perform all statistical analysis. Independent sample t-tests were performed to compare group sample means. P-values < 0.05 were considered significant.

Results: The titanium plate with screws repair demonstrated a significantly higher load at break across all metacarpals (avg=91.745 \pm 12.575$)$. There was no significant difference between the load at break for the cyanoacrylate glue (avg=39.855 \pm 8.105$)$ and the cyanoacrylate glue mixed with DBM groups (avg=28.664 \pm 8.375$)$. The 4th metacarpal produced the lowest load at break for the titanium plate with screws repair and the cyanoacrylate repair while the 5 th metacarpal presented the lowest load at break for the cyanoacrylate mixed with DBM repair.

Conclusions: The addition of DBM to cyanoacrylate does not significantly compromise the adhesive strength of cyanoacrylate. Titanium plates offer the strongest repair of the three groups. When bone fragments are too small to attach plates, the cyanoacrylate and DBM mixture creates a putty allowing easy realignment of the fragments while maintaining the strength of cyanoacrylate. This study was not conducive to examining DBM's osteoinductive nor osteoconductive properties since all tests were done ex vivo. Further research should examine repairs over time using cyanoacrylate mixed with DBM in vivo.
\end{abstract}

\section{Introduction}

Metacarpal fractures comprise roughly $40 \%$ of hand fractures [1]. When the majority of people refer to a "broken hand", most likely they are referring to a fracture of one of the metacarpal bones. Injury can occur in the neck, shaft, or head of the metacarpals [1]. The fifth metacarpal is the most commonly injured metacarpal, usually caused by punching an object and commonly referred to as a "boxer's fracture" [2]. The fractures can be identified as oblique, transverse, spiral, or comminuted [2]. Comminuted fractures of the hand are technical challenges that surgeons have to confront. The lack of bone infrastructure complicates fixation, obligating the patient to suffer longer periods of pain, immobilization and rehabilitation. Even when surgery is an available option, the demanding effort and long operative times are substantial. Comminuted fractures most commonly occur at the metacarpal head and generally have articular involvement [2]. Current surgical interventions include Kirschner wire (K-wire), screws, or plates. For comminuted fractures, K-wire is generally considered the best option due to its low invasiveness and long-term function [1]. K-wire, however, can lead to infection and requires a follow-up procedure with the patient to be removed. Bio-absorbable plates have shown promising results in cadaveric studies, but due to concerns about foreign body reactions after implantation need to be further studied before being used in patients [1].

Adhesives provide an alternative solution that is minimally invasive, offers strong fixation, and preserves soft tissue, blood supply, and trophic factors of bone [3]. Cyanoacrylate has shown recent promise as a strong adhesive that does not cause significant inflammatory responses when implanted [3]. Its use is very popular amongst surgeons as a common tool to close wounds and also as a final dressing. Cyanoacrylate's various other uses include: dental adhesives, temporary repair of corneal perforations, drug carriers, controlling variceal bleeding, controlling hemorrhages, and non-load bearing cranio-facial skeletal procedures $[3,4]$. Cyanoacrylate offers the advantages of quick one-step application without the need for external energy, easy storage, and strong adhesions to multiple surfaces even in wet conditions [4]. Further testing needs to be completed to observe its efficacy for other possible injuries. Demineralized Bone Matrix (DBM) has gained attention due to its

${ }^{*}$ Correspondence to: Wesley P Thayer, Department of Plastic Surgery, Vanderbilt University Medical Center, 1161 21st Ave S., MCN D4207, Nashville, TN 37232-2345, USA, Tel: 615-936-3759; Fax: 615-936-0167; E-mail: wesley.thayer@vanderbilt.edu

Received: June 22, 2018; Accepted: July 13, 2018; Published: July 18, 2018 
easy availability along with its osteoconductive and osteoinductive properties [5]. Effectiveness of DBM can vary widely based on how it is processed, washed, and stored [5]. DBM has no risk of an immune response from the host due to its antigens being destroyed during the demineralization process [5]. Some literature recommends its use over autogenous bone grafts due to improved outcomes [6]. A combination therapy of cyanoacrylate mixed with DBM could provide a synergistic benefit for bone repair that has not been tested yet. This innovative combination adhesive needs to be tested against both cyanoacrylate by itself and against current hardware to prove its efficacy. The specific aim for this project is to test the strength of cyanoacrylate mixed with demineralized bone matrix to repair comminuted fractures in metacarpals. A novel technique is necessary to provide a simple and fast way to treat these difficult cases.

\section{Methods}

Lightly embalmed fresh cadavers were obtained under the cadaveric anatomical gift program of the Vanderbilt Anatomy Laboratory in compliance with institutional policies and under the supervision of support personnel. Any cadavers with history of osteopenia, osteoporosis, hand trauma or hand surgery were excluded from the study. From two cadavers, the second, third, fourth, and fifth metacarpals were collected from both right and left hands. All the metacarpals from one hand were put in their own designated container to ensure all four metacarpals were used for the same treatment. The metacarpals were transected with a hand saw at each methaphysis at a 30-degree angle to achieve a comminuted fracture (Figure 1). The metacarpals from each hand were divided into three groups. The first group, denominated Plates/screws, was repaired using titanium plates with one $2.0 \mathrm{~mm}$ diameter screw $4 \mathrm{~mm}$ long, that was placed unicortically in each bone fragment (Figure 2). The next group, the Glue-only group was repaired using only commercially available ethyl-cyanoacrylate glue on the surface of each bone fragment. Once fixated, a wax paper wrap was placed around the shaft of the bone to ensure proper alignment remained during testing. (Figure 3 ) The last group, the Glue/DBM was repaired using equal parts demineralized bone matrix and ethyl-cyanoacrylate glue on the bone fragments. This group was also wrapped with the wax paper to retain proper alignment after repair. An Instron 5542 machine was used to calculate the load and energy at the break. In order to attach the bones to the Instron machine without severely damaging the bone, $1 / 2$ " diameter wood blocks were clamped into each loading cell with $1 / 16$ " diameter holes through the bottom of the blocks. 1/16" diameter holes were drilled

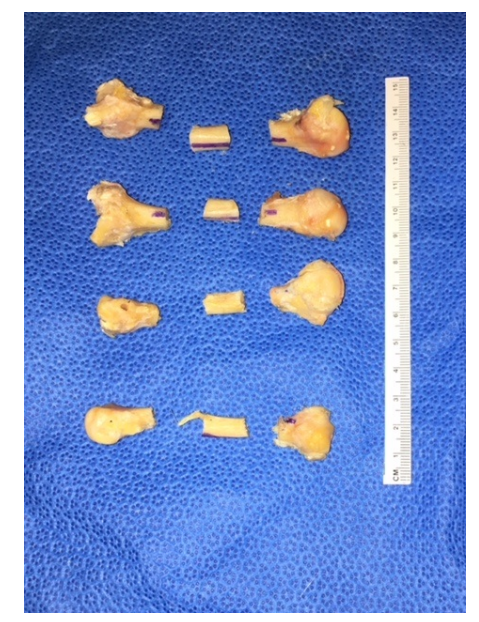

Figure 1. Comminuted fractures in metacarpals

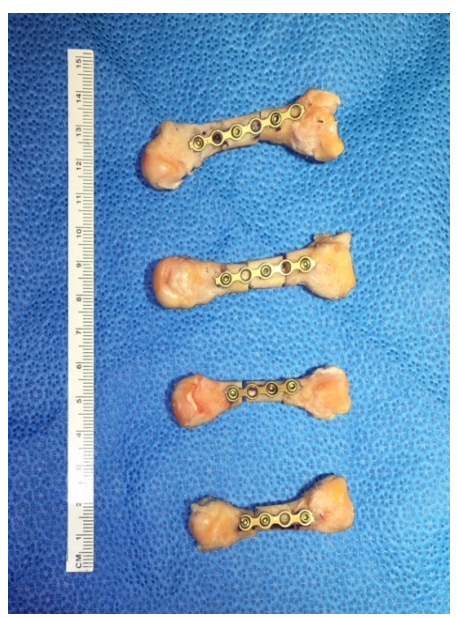

Figure 2. Plate with screws repair

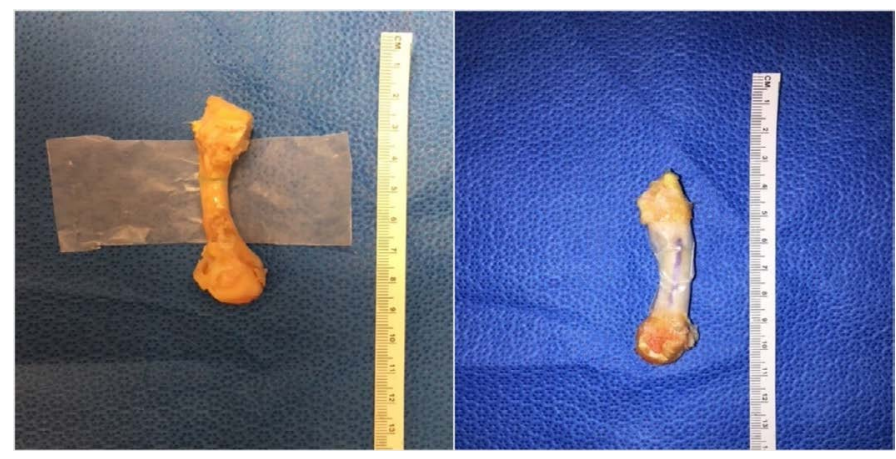

Figure 3. Bone Repaired with Glue and Demineralized Bone Matrix before (left) and after (right) wrap

through the head and base of each metacarpal and a stainless-steel wire was run through both the wood and the bone then tied at the ends to avoid slippage. (Figure 4) Once the metacarpal was properly placed in the Instron, a tension test was performed which pulled the bone longitudinally at a rate of $2 \mathrm{~mm}$ per second until break.

IBM SPSS Statistics 24 was used to perform all statistical analysis. Independent sample t-tests were performed to compare group sample means. P-values $<0.05$ were considered significant.

\section{Results}

The bones treated with Glue-only had an average load at break of $39.85 \mathrm{~N}$ and the metacarpals treated with Glue/DBM had an average load at break of $28.66 \mathrm{~N}$ (Figure 5), with no statistical significance between the two groups $(\mathrm{p}=0.37)$. The Plate/screws group had an average load at break of $91.74 \mathrm{~N}$ (Figure 5), when compared to the Glue-only and the Glue/DBM, the difference was statistically significant $(\mathrm{p}=0.004$ and $\mathrm{p}=0.001$, respectively).

The load at break of each individual metacarpal was compared between groups. The second metacarpal was the strongest across all groups (Table 1, Figure 6). The weakest bone was the fourth metacarpal in the Glue-only and Plates/screws groups (Table 1, Figure 6). The fifth metacarpal was weakest in the Glue/DBM group (Table 1, Figure 6).

\section{Discussion}

Comminuted fractures need a more effective and surgically simple form of repair. Cyanoacrylate has yet to be tested on bone repair outside of maxillofacial surgeries [7]. Demineralized bone matrix has 


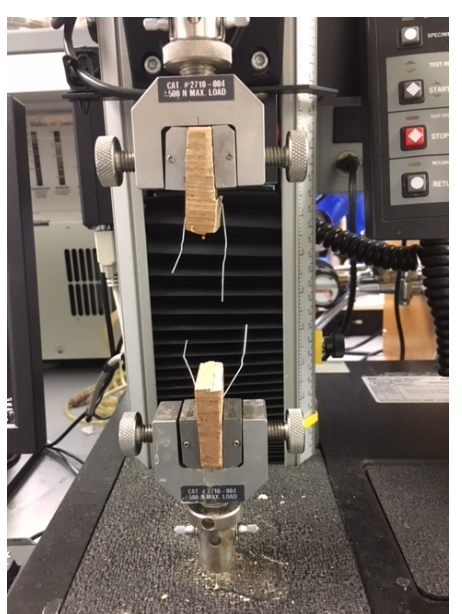

Figure 4. Instron with customized grips

\section{Repair Comparison Load at Break}

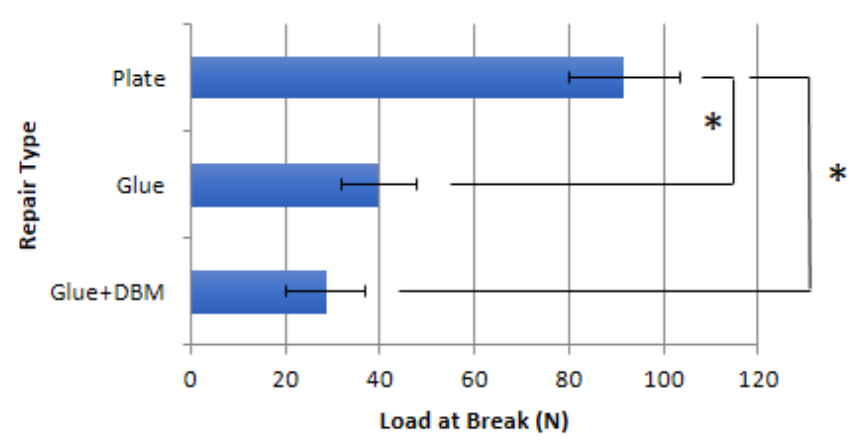

Figure 5. Average Load at Break between Repair Groups (N)

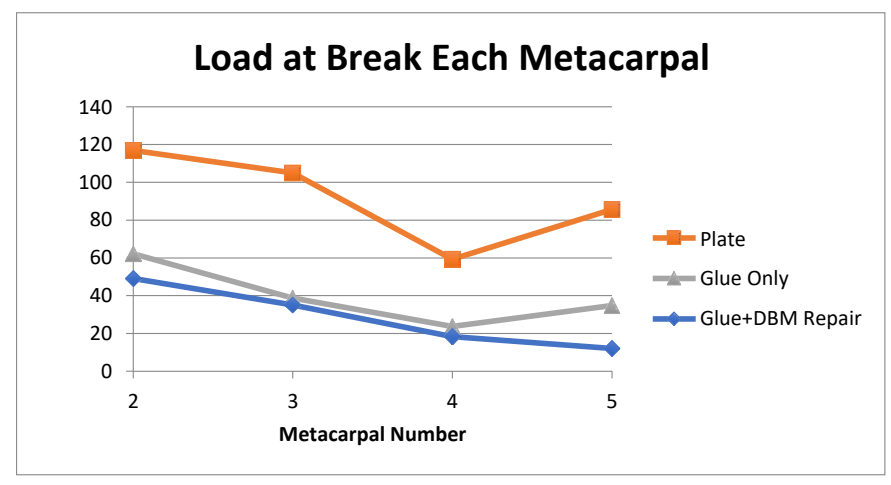

Figure 6. Load at break for each metacarpal between repair groups

Table 1. Load at break (N) of each metacarpal across repair groups

\begin{tabular}{|c|c|c|c|}
\hline Metacarpal \# & Glue+DBM & Glue & Plates/Screws \\
\hline 5 th & 12.06545 & 34.77707 & 116.924875 \\
\hline 4th & 18.35927 & 23.69474 & 105.021705 \\
\hline 3rd & 35.11009 & 38.72836 & 59.328725 \\
\hline 2nd & 49.1219 & 62.21903 & 85.702895 \\
\hline AVG & 28.6641775 & 39.8548 & 91.74455 \\
\hline SD & 16.7508912 & 16.210681 & 25.1503407 \\
\hline SEM & 8.37544562 & 8.10534048 & 12.5751704 \\
\hline
\end{tabular}

shown promise in filling in defects in bone [8]. There has yet to be an effective glue combination with demineralized bone matrix without severely compromising the strength of the bonding adhesive [8]. This combination of cyanoacrylate glue and demineralized bone matrix could provide the quick and simple repair needed for comminuted fractures. Both have individually shown promise in fields of orthopedics and dentistry with new and exciting discoveries still being made concerning the properties of each $[9,10]$.

The data indicates that demineralized bone matrix can be mixed with cyanoacrylate without significantly affecting the maximum load that the repair can withstand. It is important to note the subjective ease of repair that the Glue/DBX mixture allows over just cyanoacrylate by itself. The DBX acts like a putty that provides surface area for the glue to hold each bone fragment together. With cyanoacrylate by itself, if the bone fragment surfaces were not perfectly in contact with each other then the glue would not hold. This is an important observation when considering future clinical application since comminuted fractures will not be as clean and precise as the fractures recreated in this experiment. The titanium plate intervention was by far the strongest group, and should be recommended as the standard of care for fractures of larger bone. The real dilemma remains when bone fragments after a fracture are too small to use plates.

One peculiar finding was that for the Plate/screw and Glue-only groups, the fourth metacarpal withstood less force than the fifth metacarpal. One would expect that since the fifth metacarpal is the smallest bone and the most commonly fractured, its repair would be the weakest out of the four metacarpals tested. At first we thought we had simply mistook the fourth metacarpal for the fifth, but when the same results appeared for both plate and glue groups, we proposed different solutions. The fourth metacarpal in men is significantly narrower than the fifth metacarpal [11] This reduction in surface area for the glue to hold is the simplest explanation to why the fourth metacarpal was weaker than the fifth. Another proposed theory is that since the fifth metacarpal was more fragile, it was easier to fracture and as a result had a cleaner fracture than the fourth metacarpal. Due to their similarity in size, this made the repair on the cleaner cut stronger with both the glue only and the plates with screws. Conversely, in the Glue/DBM group, the demineralized bone matrix was able to compensate for the uneven surfaces by creating a uniform surface over the fragments. This intervention by itself increased improved the repair by increasing the surface area in contact with the adhesive. Accordingly, the Glue/DBX's strength decreased uniformly with size of the metacarpal.

The largest limitation of our study is that the form of cyanoacrylate we used was ethyl-cyanoacrylate. This has a shorter side chain than other commonly used cyanoacrylate products. Ethyl-cyanoacrylate has been shown to exhibit toxicity in clinical use [4]. Before in vivo testing, stability of butyl-cyanoacrylate or octyl-cyanoacrylate mixed with demineralized bone matrix must be tested. Future studies could compare the three types of cyanoacrylate to see which could provide the strongest repair with the least likelihood for immunological response. Another limitation is that we are not able to assess the demineralized bone matrix's osteoinductive or osteoconductive properties since this study was ex vivo. These limitations should be focused on for future aims of our studies.

\section{Conclusion}

Comminuted fractures are painful and difficult for both patients and surgeons. A strong, quickly acting adhesive should be able to improve outcomes and cut down on surgical times. The strength 
and speed of cyanoacrylate combined with the osteoinductive and osteoconductive properties of demineralized bone matrix provides an ideal treatment for comminuted fractures. Further testing should experiment with different sized cyanoacrylate molecules (butyl or octyl) to see if demineralized bone matrix is compatible with variations of cyanoacrylate with longer side chains.

\section{Acknowledgement}

This study was supported by Vanderbilt University Medical Center Department of Plastic Surgery funds.

\section{References}

1. Haughton D, Jordan D, Malahias M, Hindocha S, Khan W (2012) Principles of hand fracture management. Open Orthop J 6: 43-53. [Crossref]

2. Kollitz KM, Hammert WC, Vedder NB, Huang JI (2014) Metacarpal fractures: treatment and complications. Hand (N Y) 9: 16-23. [Crossref]

3. Akcal MA, Poyanli O, Unay K, Esenkaya I, Gokcen B, et al. (2014) Effect of N-butyl cyanoacrylate on fracture healing in segmental rat tibia fracture model. J Orthop Surg Res 9: 76. [Crossref]

4. Korde JM, Kandasubramanian B (2018) Biocompatible alkyl cyanoacrylates and their derivatives as bio-adhesives. Biomater Sci 6: 1691-1711. [Crossref]
5. Drosos GI, Touzopoulos P, Ververidis A, Tilkeridis K, Kazakos K (2015) Use of demineralized bone matrix in the extremities. World $J$ Orthop 6: 269-277. [Crossref]

6. Pieske O, Wittmann A, Zaspel J, Löffler T, Rubenbauer B, et al. (2009) Autologous bone graft versus demineralized bone matrix in internal fixation of ununited long bones. J Trauma Manag Outcomes 3: 11. [Crossref]

7. Habib A, Mehanna A, Medra A (2013) Cyanoacrylate: a handy tissue glue in maxillofacial surgery: our experience in alexandria, egypt. J Maxillofac Oral Surg 12: 243-247. [Crossref]

8. Silva LCFD, Porto GG, Andrade ESS, Laureano Filho JR (2018) Demineralized bone matrix and calcium-phosphate cement in bone regeneration in rats. Acta Cir Bras 33: 354-361. [Crossref]

9. Pietrzak WS (2018) Stranger Things: A Whimsical Account of a Demineralized Bone Matrix Study with Unexpected Results. J Craniofac Surg 29: 1107-1109. [Crossref]

10. Bissacco D, Stegher S, Calliari FM, Viani MP (2018) Saphenous vein ablation with a new cyanoacrylate glue device: a systematic review on 1000 cases. Minim Invasive Ther Allied Technol. [Crossref]

11. Soong M, Got C, Katarincic J (2010) Ring and little finger metacarpal fractures: mechanisms, locations, and radiographic parameters. J Hand Surg Am 35: 1256-1259. [Crossref]

Copyright: (C2018 Stephanides M. This is an open-access article distributed under the terms of the Creative Commons Attribution License, which permits unrestricted use, distribution, and reproduction in any medium, provided the original author and source are credited. 